\title{
Applications of gold nanoparticles in cancer nanotechnology
}

\author{
Weibo Cai ${ }^{1,2}$ \\ Ting $\mathrm{Gao}^{3}$ \\ Hao Hong' \\ Jiangtao Sun' \\ 'Departments of Radiology \\ and Medical Physics, School \\ of Medicine and Public Health, \\ University of Wisconsin - Madison, \\ Madison, Wisconsin, USA; ${ }^{2}$ University \\ of Wisconsin Paul P. Carbone \\ Comprehensive Cancer Center, \\ Madison, Wisconsin, USA; ${ }^{3}$ Tyco \\ Electronics Corporation, 306 \\ Constitution Drive, Menlo Park, \\ California, USA
}

\begin{abstract}
It has been almost 4 decades since the "war on cancer" was declared. It is now generally believed that personalized medicine is the future for cancer patient management. Possessing unprecedented potential for early detection, accurate diagnosis, and personalized treatment of cancer, nanoparticles have been extensively studied over the last decade. In this review, we will summarize the current state-of-the-art of gold nanoparticles in biomedical applications targeting cancer. Gold nanospheres, nanorods, nanoshells, nanocages, and surface enhanced Raman scattering nanoparticles will be discussed in detail regarding their uses in in vitro assays, ex vivo and in vivo imaging, cancer therapy, and drug delivery. Multifunctionality is the key feature of nanoparticle-based agents. Targeting ligands, imaging labels, therapeutic drugs, and other functionalities can all be integrated to allow for targeted molecular imaging and molecular therapy of cancer. Big strides have been made and many proof-of-principle studies have been successfully performed. The future looks brighter than ever yet many hurdles remain to be conquered. A multifunctional platform based on gold nanoparticles, with multiple receptor targeting, multimodality imaging, and multiple therapeutic entities, holds the promise for a "magic gold bullet" against cancer.
\end{abstract}

Keywords: gold nanoparticles, cancer, nanotechnology, optical imaging, nanomedicine, molecular therapy

\section{Introduction}

Cancer is the third leading cause of death (after heart disease and stroke) in developed countries and the second leading cause of death (after heart disease) in the United States (see http://www.cdc.gov). Studies have shown that there were 10 million new cases, 6 million deaths, and 22 million people living with cancer worldwide in the year 2000 (Parkin 2001). These numbers represent an increase of about $22 \%$ in incidence and mortality from that of the year 1990 (Parkin et al 1999; Pisani et al 1999). It is projected that the number of new cases of all cancers worldwide will be 12.3 and 15.4 million in the year 2010 and 2020, respectively (Parkin 2001). In 2008, a total of $1,437,180$ new cancer cases and 565,650 cancer deaths were estimated to occur in the United States alone (Jemal et al 2008).

Nanotechnology, an interdisciplinary research field involving chemistry, engineering, biology, and medicine, has great potential for early detection, accurate diagnosis, and personalized treatment of cancer (Cai and Chen 2007). Nanoparticles are typically smaller than several hundred nanometers in size, comparable to large biological molecules such as enzymes, receptors, and antibodies. With the size of about one hundred to ten thousand times smaller than human cells, these nanoparticles can offer unprecedented interactions with biomolecules both on the surface of and 
inside the cells, which may revolutionize cancer diagnosis and treatment. The most well-studied nanoparticles include quantum dots (Cai et al 2006, 2007b), carbon nanotubes (Liu et al 2007b), paramagnetic nanoparticles (Thorek et al 2006), liposomes (Park et al 2004), gold nanoparticles (Huang et al 2007b), and many others (Ferrari 2005; Grodzinski et al 2006) (Figure 1).

Over the last decade, there have been many nanotechnology centers established worldwide (Kawasaki and Player 2005; Horton and Khan 2006). In the United States alone, more than six billion dollars have been invested in nanotechnology research and more than sixty centers, networks, and facilities, funded by various agencies, are in operation or soon to open (Thayer 2007). After establishing an interdisciplinary nanotechnology workforce, it is expected that nanotechnology will mature into a clinically useful field in the near future.

One of the major applications of nanotechnology is in biomedicine. Nanoparticles can be engineered as nanoplatforms for effective and targeted delivery of drugs and imaging labels by overcoming the many biological, biophysical, and biomedical barriers. For in vitro and ex vivo applications, the advantages of state-of-the-art nanodevices (eg, nanochips and nanosensors) over traditional assay methods are obvious (Grodzinski et al 2006; Sahoo et al 2007). However, several barriers exist for in vivo applications in preclinical and

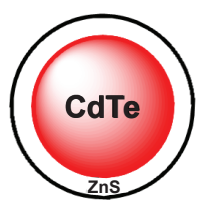

Quantum dot

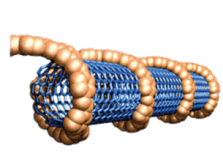

Nanotube

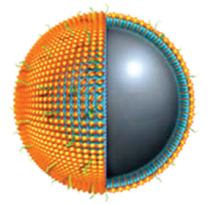

Perfluorocarbon

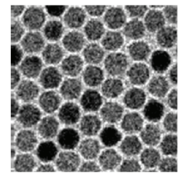

Iron oxide

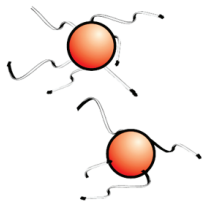

Gold

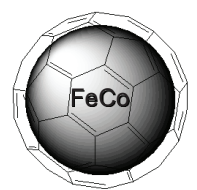

FeCo

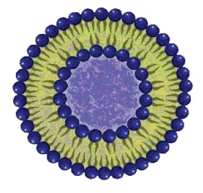

Liposome

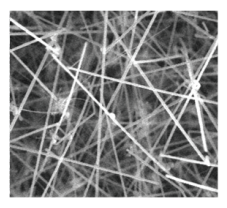

Nanowire

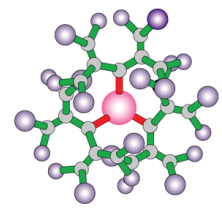

Dendrimer
Figure I Many nanoparticles have been investigated for biomedical applications targeting cancer. potentially clinical use of nanotechnology, among which are the biocompatibility, in vivo kinetics, tumor targeting efficacy, acute and chronic toxicity, ability to escape the reticuloendothelial system (RES), and cost-effectiveness (Cai and Chen 2007, 2008). In this review, we will summarize the current state-of-the-art of gold nanoparticles in biomedical applications.

\section{Synthesis of gold nanoparticles}

There are many subtypes of gold nanoparticles based on the size, shape, and physical properties (Figure 2). The earliest studied gold nanoparticles are gold nanospheres (although not exactly spherical in a strict sense). Subsequently, nanorods, nanoshells, and nanocages have all been reported. Another type of gold-based nanoparticles, with excellent surfaceenhanced Raman scattering properties (termed "SERS nanoparticles"), will also be discussed in this review. In the following text, the term "gold nanoparticle(s)" will refer to a collection of all these subtypes and the subtype of gold nanoparticles used in each study will be specified whenever possible. With continued development in the synthesis techniques over the last two decades, most of these gold nanoparticles can now be produced with well-controlled size distribution, sometimes with stunning precision (eg, nanocages).

\section{Gold nanospheres}

Gold nanospheres (also known as gold colloids) of $2 \mathrm{~nm}$ to over $100 \mathrm{~nm}$ in diameter can be synthesized by controlled reduction of an aqueous $\mathrm{HAuCl}_{4}$ solution using different reducing agents under varying conditions. The most commonly used reducing agent is citrate, which can produce nearly monodisperse gold nanospheres (Turkevich et al 1951; Frens 1973). The size of the nanospheres can be controlled by varying the citrate/gold ratio. Generally, smaller amount of citrate will yield larger nanospheres. The major limitations of this method are the low yield and the restriction of using water as the solvent.

A two-phase method, inspired by the two-phase system used by Faraday in 1857, capable of producing thermally and air stable gold nanospheres of reduced dispersity and well-controlled size (usually $<10 \mathrm{~nm}$ in diameter) was reported in 1993 (Giersig and Mulvaney 1993). This technique was later improved through the use of a phasetransfer reagent such as tetraoctylammonium bromide (Brust et al 1994). Again, the thiol/gold molar ratios can affect the average size of the nanospheres. Larger thiol/gold ratios and 


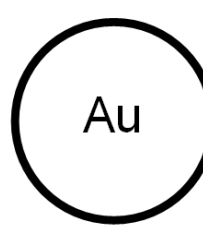

Sphere

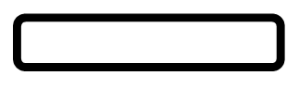

Rod

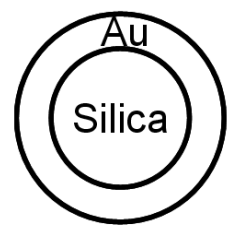

Shell

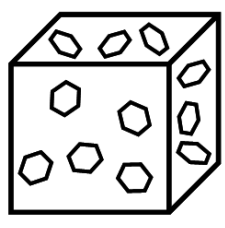

Cage

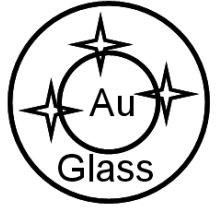

SERS

Figure 2 Different types of gold nanoparticles.

faster addition of the reductant in cooled solutions will yield smaller and more monodispersed gold nanospheres.

Several other methods have been investigated for gold nanosphere synthesis such as the use of other reductants or ligands (Leff et al 1996; Weare et al 2000; Hiramatsu and Osterloh 2004). There are a number of literature reports on the use of dendrimers as templates or stabilizers for gold nanosphere preparation (Esumi et al 1998; Garcia et al 1999; Manna et al 2001; Kim et al 2004; Scott et al 2005; Shi et al $2006,2007 b, 2008)$. Biocompatible block copolymers have been employed for the synthesis of sterically stabilized gold nanospheres in aqueous solution (Yuan et al 2006). The size and shape of the gold nanospheres could be readily controlled by tuning the synthesis parameters such as the block composition, and the relative/absolute concentrations of the block copolymer and $\mathrm{HAuCl}_{4}$. Growth of gold nanospheres in human cells has also been reported (Anshup et al 2005).

Typically, gold nanospheres display a single absorption peak in the visible range between $510 \mathrm{~nm}$ and $550 \mathrm{~nm}$. With increasing particle size, the absorption peak shifts to a longer wavelength and the width of the absorption spectra is related to the size distribution range. Many other types of gold nanoparticles with different size/shape, such as nanorods, nanoshells, and nanocages, have been explored to obtain optical properties suitable for biomedical applications.

\section{Gold nanorods}

The synthesis of gold nanorods has been reported using a wide variety of strategies. Gold nanorods are typically synthesized using the template method, based on the electrochemical deposition of gold within the pores of nanoporous polycarbonate or alumina template membranes (Martin 1994; van der Zande et al 1997). The diameter of the gold nanorod is determined by the pore diameter of the template membrane, while the length of the nanorod can be controlled through the amount of gold deposited within the pores of the membrane. A fundamental disadvantage of this method is the low yield since only one monolayer of nanorods is prepared. Formation of gold nanorods through electrochemical synthesis has also been reported (Reetz and Helbig 1994; Yu et al 1997; Chang et al 1999). In this approach, many experimental parameters can determine the length of the nanorod thus affecting its aspect ratio (defined as the length divided by the width).

Seed-mediated synthesis, perhaps the most wellestablished method for gold nanorod preparation, can provide higher aspect ratios than those prepared by other methods (Jana et al 2001b; Busbee et al 2003). Usually gold seeds are made by chemical reduction of a gold salt with a strong reducing agent such as $\mathrm{NaBH}_{4}$. These seeds, serving as the nucleation sites for nanorods, are then added to a growth solution of gold salt with a weak reducing agent such as ascorbic acid and hexadecyltrimethylammonium bromide. The aspect ratios of the gold nanorods can be controlled by varying the amount of gold seeds with respect to the gold precursor. Moreover, gold nanorods can be produced in quantitative yield with the addition of $\mathrm{AgNO}_{3}$ (Jana et al 2001a, 2002). Besides the methods mentioned above, several other approaches have also been investigated for the fabrication of gold nanorods, including bio-reduction (Canizal et al 2001), growth on mica surface (Mieszawska and Zamborini 2005), and photochemical synthesis (Kim et al 2002).

\section{Gold nanoshells}

Optical imaging, include those that uses gold nanoparticles as the contrast agents, has very limited applications in human studies. However, in the near-infrared region (NIR; 700-900 nm), the absorbance of all biomolecules reaches minimum which provides a relatively clear window for optical imaging (Frangioni 2003). By varying the composition and dimensions of the layers, gold nanoshells can be designed and fabricated with surface plasmon resonance (SPR) peaks ranging from the visible to the NIR region (Oldenburg et al 1999b). For a given composition of gold nanoshell, the SPR peak can be tuned by changing the ratio of the core size to its shell thickness.

Gold nanoshells with SPR peaks in the NIR region can be prepared by coating silica or polymer beads with gold shells of variable thickness (Caruso et al 2001; 
Oldenburg et al 1998). Silica cores are grown using the Stöber process, the basic reduction of tetraethyl orthosilicate in ethanol. To coat the silica nanoparticles with gold in an aqueous environment, a seeded growth technique is typically used. Small gold nanospheres (2-4 $\mathrm{nm}$ in diameter) can be attached to the silica core using an amine-terminated silane as a liner molecule, allowing additional gold to be reduced until the seed particles coalesced into a complete shell (Oldenburg et al 1999a). The diameter of the gold nanoshell is largely determined by the diameter of the silica core, and the shell thickness can be controlled through the amount of gold deposited on the surface of the core.

Gold nanoshells have also been synthesized via in situ gold nanoparticle formation using thermosensitive core-shell particles as the template (Suzuki and Kawaguchi 2005). The use of microgel as the core offers significantly reduced particle aggregation, as well as thickness control of the gold nanoshells using electroless gold plating. In one study, a virus scaffold has been used to assemble gold nanoshells (Radloff et al 2005). This approach may potentially provide cores with a narrower size distribution and smaller diameters $(<80 \mathrm{~nm})$ than those of silica.

\section{Gold nanocages}

Gold nanocages with controllable pores on the surface have been synthesized via galvanic replacement reaction between truncated silver nanocubes and aqueous $\mathrm{HAuCl}_{4}$ (Chen et al 2006). Silver nanostructures with controlled morphologies can be generated through polyol reduction, where $\mathrm{AgNO}_{3}$ is reduced by ethylene glycol to generate silver atoms and then nanocrystals or seeds. Subsequent addition of silver atoms to the seeds produces the desired nanostructures through controlling the silver seed crystalline structures in the presence of poly(vinylpyrrolidone), a polymer that is capable of selectively binding to the (100) surface. The silver nanostructures, used as a sacrificial template, can then be transformed into gold nanostructures with hollow interiors via the galvanic replacement (Chen et al 2005, 2006). The dimension and wall thickness of the resultant gold nanocages could be readily controlled, to very high precision, by adjusting the molar ratio of silver to $\mathrm{HAuCl}_{4}$.

\section{SERS nanoparticles}

SERS is an optical technique that offers many advantages over traditional technologies, such as fluorescence and chemiluminescence, including better sensitivity, high levels of multiplexing, robustness, and superior performance in blood and other biological matrices (Sha et al 2007;
Hering et al 2008). In a pioneering report, gold nanospheres ( $\sim 13 \mathrm{~nm}$ in diameter) modified with Cy3-labeled, alkylthiolcapped oligonucleotide strands were used as probes to monitor the presence of specific target DNA strands (Cao et al 2002). The Cy 3 group was chosen as the Raman label because of its large Raman cross-section.

Subsequently, several other reports have also used SERS nanoparticles. In one study, gold nanospheres (60 nm in diameter) were encoded with a Raman reporter and stabilized with a layer of thiolated polyethylene glycol (PEG) (Qian et al 2008). Another type of SERS nanoparticle is composed of a gold core, a Raman-active molecular layer, and a silica coating (Keren et al 2008). The silica coating can ensure physical robustness, inertness to various environmental conditions, and simple surface modification via silica chemistry. The thiol groups that were subsequently introduced onto the silica shell can be conjugated with maleimideactivated PEG chains for improved biocompatibility.

\section{Applications of nanoparticles}

Nanotechnology has been an extremely hot topic over the last decade. A simple search of "Nano" in PubMed returned more than 6000 publications. Two major areas of nanoparticle applications are material science and biomedicine. Big strides have been made in the material science arena. The fact that electronics are getting faster, better, and smaller each month is a clear and strong evidence for such achievement. However, applications of nanoparticles in the biomedical field have not fulfilled the expectations. Very few nanoparticle-based agents are in clinical testing or commercialized for cancer diagnosis or treatment, and most of them are based on liposomes which were developed several decades ago. There is still a long way to go before nanotechnology can truly revolutionize patient care as many have hoped it would. Next, we will summarize the progress to date regarding the use of gold nanoparticles for biomedical applications.

\section{Biomedical applications of gold nanoparticles}

Cancer nanotechnology is an interdisciplinary area with broad potential applications in fighting cancer, including molecular imaging, molecular diagnosis, targeted therapy, and bioinformatics. The continued development of cancer nanotechnology holds the promise for personalized oncology in which genetic and protein biomarkers can be used to diagnose and treat cancer based on the molecular profile of each individual patient. Gold nanoparticles have been investigated 
in diverse areas such as in vitro assays, in vitro and in vivo imaging, cancer therapy, and drug delivery.

\section{In vitro assays}

Oligonucleotide-capped gold nanoparticles have been reported for polynucleotide or protein (such as p53, a tumor suppressor gene) detection using various detection/characterization methods such as atomic force microscopy (AFM) (Han et al 2000; Jin et al 2007), gel electrophoresis (Qin and Yung 2007), scanometric assay (Son and Lee 2007), chronocoulometry (Zhang et al 2007), amplified voltammetric detection (Wang et al 2008a), SPR imaging (Li et al 2006), and Raman spectroscopy (Cao et al 2002). In some reports, picomolar even femtomolar concentrations of DNA targets have been detected. Bifunctional DNA-based adsorbate molecules have been evaluated as molecular rulers, based on the SERS signals that vary independently in intensity as a function of the distance from the gold nanoshell surface (Lal et al 2006).

Gold nanoparticles have also been employed for many other applications such as immunoassay (Hirsch et al 2003a, 2005; Liu et al 2008), protein assay (Tang et al 2007), timeof-flight secondary ion mass spectrometry (Kim et al 2006), capillary electrophoresis (Tseng et al 2005), and detection of cancer cells (Kah et al 2007; Medley et al 2008). In one report, dynamic light scattering (DLS) enabled quantitative estimation of the concentration of intravenously injected gold nanoshells in mouse blood (Xie et al 2007). This technique may also be applicable towards estimating the circulation life time of other solid nanoparticles. Gold nanoshells functionalized with a $\mathrm{pH}$-sensitive SERS reporter molecule, 4-mercaptopyridine, were shown to be responsive to the $\mathrm{pH}$ of the surrounding media within the range of 3 to 7 (Jensen et al 2007). Another study has evaluated the use of gold nanoshells as optical biosensors for real-time detection of streptavidinbiotin interactions in diluted human blood (Wang et al 2008b). However, both the sensitivity $(\sim 3 \mu \mathrm{g} / \mathrm{mL})$ and the dynamic range $(3-50 \mu \mathrm{g} / \mathrm{mL})$ were very poor.

In many cases, literature reports have mixed findings. It will be ideal if different assays can be compared side-by-side using the same model system, which can significantly help in deciding which assays are the best candidates for potential clinical testing (Figure 3). The National Cancer Institute (NCI) alliance for nanotechnology in cancer has required each of its funded centers to test their newly developed nanosensors using the same standard samples, which is expected to readily identify which new sensors truly stand out from the large pool of new molecular sensors. Extending a similar standard to a much broader range of research labs across the country would be highly beneficial to cancer patients. Choosing the right candidate at an early stage not only saves precious time, but also can dramatically reduce the cost for new assay development.

\section{Cell and phantom imaging}

To date, most imaging studies using gold nanoparticles were carried out in cell culture. The versatile optical properties of gold nanoparticles have enabled optical imaging of cells and phantoms with a wide variety of contrast mechanisms (Figure 4). Functional cellular imaging around single molecules has been reported, taking advantage of the enhanced second harmonic signal by antibodyconjugated gold nanospheres (Peleg et al 1999). Subsequently, many other studies have been reported which employed

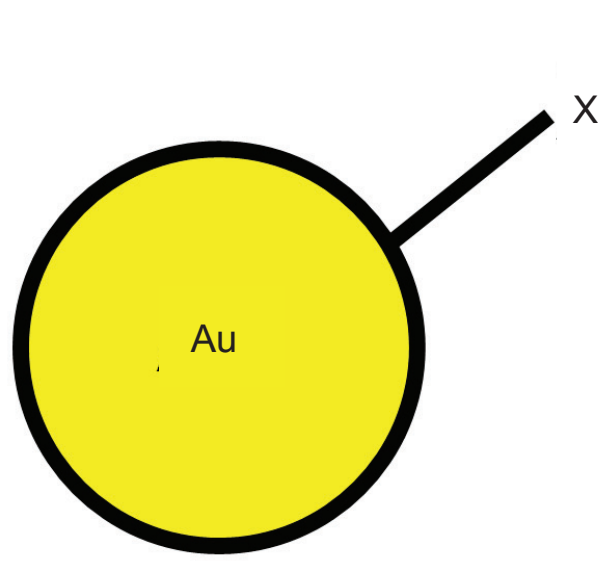

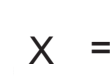
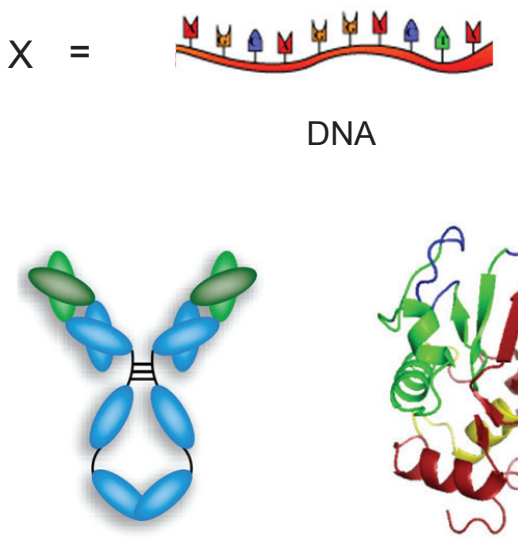

Antibody

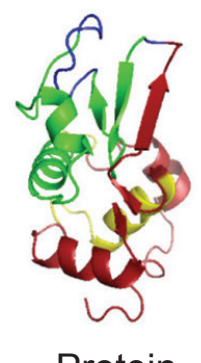

Protein

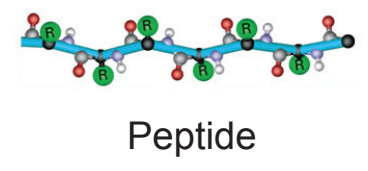

Peptide

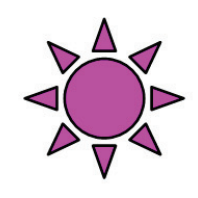

Organic dye

Figure 3 Gold nanoparticles have been modified with various molecules for in vitro assays. 


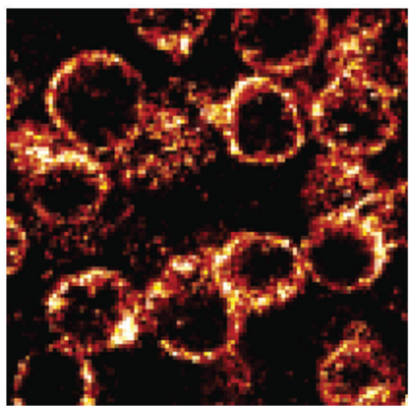

Two-photon luminescence

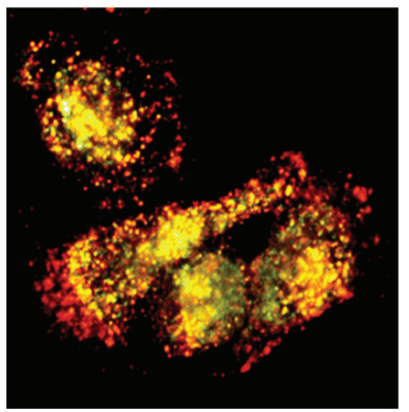

Dark-field light scattering

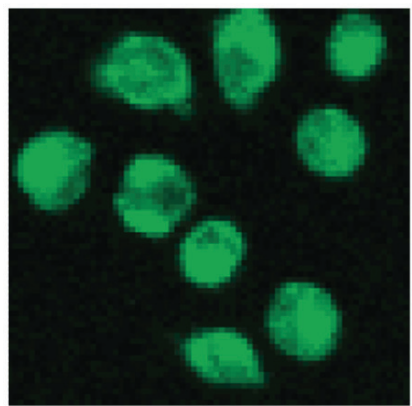

Confocal microscopy

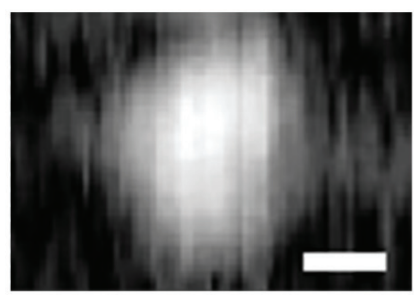

Photoacoustic tomography

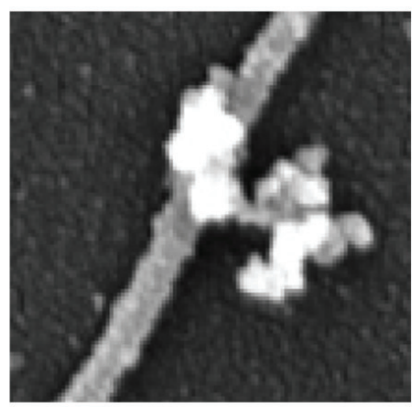

Scanning electron microscopy

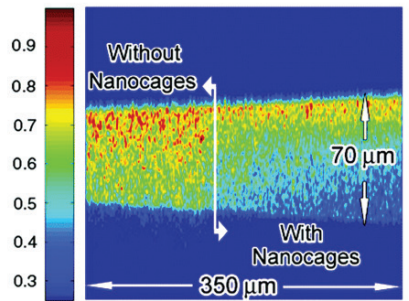

Optical coherence tomography

Figure 4 Gold nanoparticles have been investigated for cell and phantom imaging using various techniques. Adapted from Chen et al 2005; Yang et al 2005; de la Fuente et al 2006; Durr et al 2007; Li et al 2007a; Oyelere et al 2007. Scale bar: I mm.

photothermal interference contrast (Cognet et al 2003), AFM (Yang et al 2005), dark-field imaging (Loo et al 2005a; Dunn and Spudich 2007), reflectance imaging (Sokolov et al 2003; Nitin et al 2007b), as well as fluorescence and scanning electron microscopy (de la Fuente et al 2006; Shi et al 2007a).

Two-photon luminescence imaging of cancer cells in a $3 \mathrm{D}$ tissue phantom down to the $75 \mu \mathrm{m}$ depth has been achieved using gold nanorods (Durr et al 2007). The signal intensity from gold nanorod-labeled cancer cells was three orders of magnitude brighter than the two-photon autofluorescence emission from unlabeled cancer cells under $760 \mathrm{~nm}$ excitation. Fluorescent dyes have been conjugated to gold nanoparticles for fluorescence imaging of cells, upon additional modification with certain targeting ligands (Nitin et al 2007a). The advantage of imaging the gold nanoparticle itself is that there is no photobleaching or blinking, which is inherent to many other fluorophores (Yao et al 2005; Li et al 2007b). However, the disadvantage is that the optical signal of gold nanoparticles may not be as strong as certain fluorescent dyes or quantum dots.

Gold nanorods have been reported for cell imaging using techniques such as dark field light SPR scattering
(Oyelere et al 2007) and photoacoustic imaging (Li et al 2007a). Photoacoustic tomography (PAT) is a hybrid imaging modality that uses light to rapidly heat elements within the tissue, which results in photoacoustic waves (generated by thermoelastic expansion) that can be detected with an ultrasonic transducer. The use of NIR-absorbing gold nanoparticles can significantly enhance the image contrast, due to the more substantial differences in optical absorption (hence stronger photoacoustic wave generation) than the endogenous tissue chromophores. With photoacoustic imaging, multiple molecular targets have been detected simultaneously using different monoclonal antibodies conjugated to two types of gold nanorod with different aspect ratios (which have peak optical absorption at different wavelengths). Photoacoustic flow cytometry was also developed for real-time detection of circulating cells labeled with gold nanorods in the vasculature of mouse ear (Zharov et al 2006a). The threshold sensitivity was estimated to be one cancer cell in the background of $10^{7}$ normal blood cells. However, the amount of gold nanorods per tumor cell was not reported.

Nanoshell-enhanced optical coherence tomography (OCT) has the potential for molecular imaging and improved 
detection of diseases (Low et al 2006). A study of OCT at $1310 \mathrm{~nm}$ in water and turbid tissue-simulating phantoms with added nanoshells were carried out to determine the sensitivity threshold for several nanoshell geometries (Agrawal et al 2006). For the best nanoshell tested, which has a core of $291 \mathrm{~nm}$ in diameter and a shell thickness of $25 \mathrm{~nm}$, a concentration of $10^{9}$ nanoshells $/ \mathrm{mL}$ was needed to produce a signal increase. Although such concentration can be achieved theoretically under optimized conditions (assuming that $1 \%$ of each cell's surface can be covered by nanoshells), in vivo targeted imaging would be extremely challenging since nanoshells of such size may never extravasate. Similar as nanoshells, gold nanocages $(<40 \mathrm{~nm}$ in dimension) with SPR peaks around $800 \mathrm{~nm}$ have also been tested for OCT imaging of phantoms (Cang et al 2005; Chen et al 2005). Bioconjugation with antibodies enabled specific targeting of certain breast cancer cells (Chen et al 2005).

Cell and phantom imaging using gold nanoparticles serves as a proof-of-principle for their potential applications in live animals or cancer patients. It is unclear how these gold nanoparticles compare to other nanoparticles suitable for optical imaging, most notably quantum dots. Although gold nanoparticles have lower toxicity than quantum dots (Kirchner et al 2005; Hauck et al 2008), the optical characteristics of quantum dots appear to be far more superior in cell-based investigations (Michalet et al 2005; Cai et al 2007b). In vivo targeted cancer imaging using nanoparticles has rarely been achieved (Sipkins et al 1998; Gao et al 2004; Cai et al 2006, 2007a), and even fewer exhibited tumor targeting efficacy that is sufficient for potential molecular imaging or molecular therapy applications in the clinical setting (Liu et al 2007b). Recently, in vivo imaging using gold nanoparticles as contrast agents has been reported.

\section{In vivo imaging}

Many paramagnetic nanoparticles have been used for magnetic resonance (MR) imaging, both preclinically and clinically (de Roos et al 1988; Thorek et al 2006). Recently, $\mathrm{Au}_{3} \mathrm{Cu}_{1}$ nanoshells were reported to be capable of enhancing the contrast of blood vessels in vivo, which suggested their potential use in MR angiography as blood-pool agents (Su et al 2007). However, due to the low sensitivity of MR imaging, a dose-dependent toxic effect of the nanoshells was observed: $17 \%$ of the mice died at a dose of $20 \mathrm{mg} / \mathrm{kg}$.

Protease sensitive self-quenched and gold nanosphere quenched probes were shown to enable visual monitoring of the activities of both proteases and protease inhibitors in vitro and in vivo (Lee et al 2008). This technique can also be applied to other proteases by using the appropriate peptide substrate as the spacer, as previously demonstrated by other studies which took advantage of the self-quenching properties of organic fluorescent dyes (Weissleder et al 1999; Bremer et al 2001; Shah et al 2004; Jaffer et al 2007).

Raman spectroscopy is the most promising imaging technique for gold nanoparticle-based contrast agents. The Raman spectra and Raman images of methylene blue molecules adsorbed as a single layer on gold nanospheres were found useful for studying the plasmon properties (Laurent et al 2005). Later, antibody conjugated gold nanorods were reported to give a Raman spectrum that is greatly enhanced, sharpened, and polarized (Huang et al 2007a). In these two reports, Raman imaging was tested in cells but not in living subjects. Recently, two groups have independently reported the in vivo targeted imaging of cancer using Raman spectroscopy and SERS nanoparticles. In the first report, it was shown that small molecule Raman reporters (such as fluorescent dyes) were stabilized by thiolated PEG and gave large optical enhancements (Qian et al 2008). When conjugated to tumor-targeting ligands, the conjugated SERS nanoparticles were able to target tumor markers such as epidermal growth factor receptor (EGFR) on human cancer cells and in xenograft tumor models. Interestingly, although the Raman spectra of the SERS nanoparticles were reported, the Raman image was not acquired for the tumor-bearing mice.

In the other study, SERS nanoparticles composed of a gold core, a Raman-active molecular layer, and a silica coating was used for Raman imaging in vivo (Figure 5) (Keren et al 2008). A minimum detection sensitivity of 8 picomolar of SERS nanoparticles was observed in a living mouse. As a proof-of-principle, in vivo multiplexed imaging of four different SERS nanoparticles was demonstrated.

Raman imaging holds significant potential as a strategy for biomedical imaging of living subjects. However, one has to keep in mind that optical imaging in mice can not be directly scaled up to in vivo imaging in human applications due to the limited tissue penetration of optical signal. In clinical settings, optical imaging (including Raman spectroscopy) is only relevant for tissues close to the surface of the skin (for example, breast imaging), tissues accessible by endoscopy (such as the esophagus and colon), and intraoperative visualization (typically image guided surgery). NIR optical imaging devices for detecting and diagnosing breast cancer have been tested in patients and the initial results are encouraging (Taroni et al 2004; Intes 2005). Multiple SERS nanoparticles with different absorption wavelengths in the 

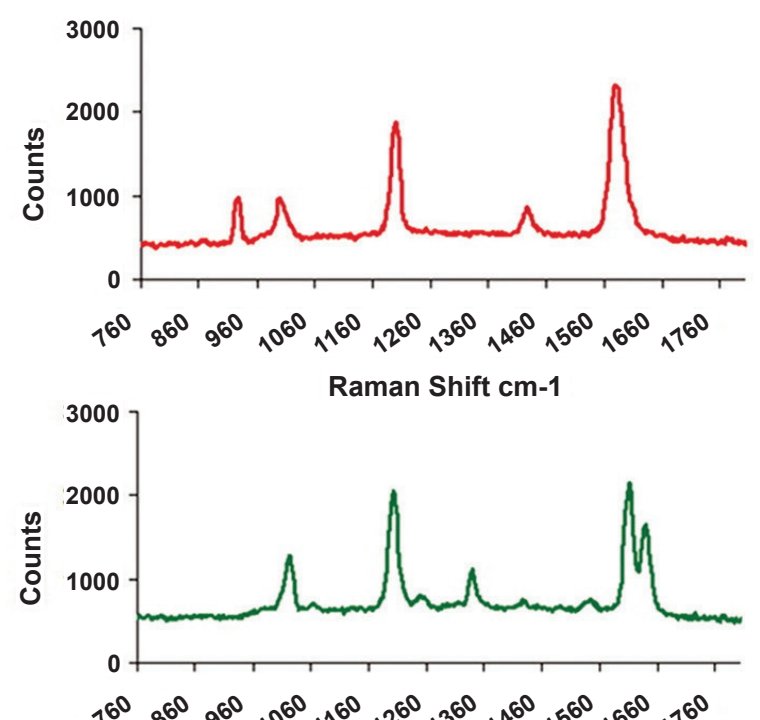

A
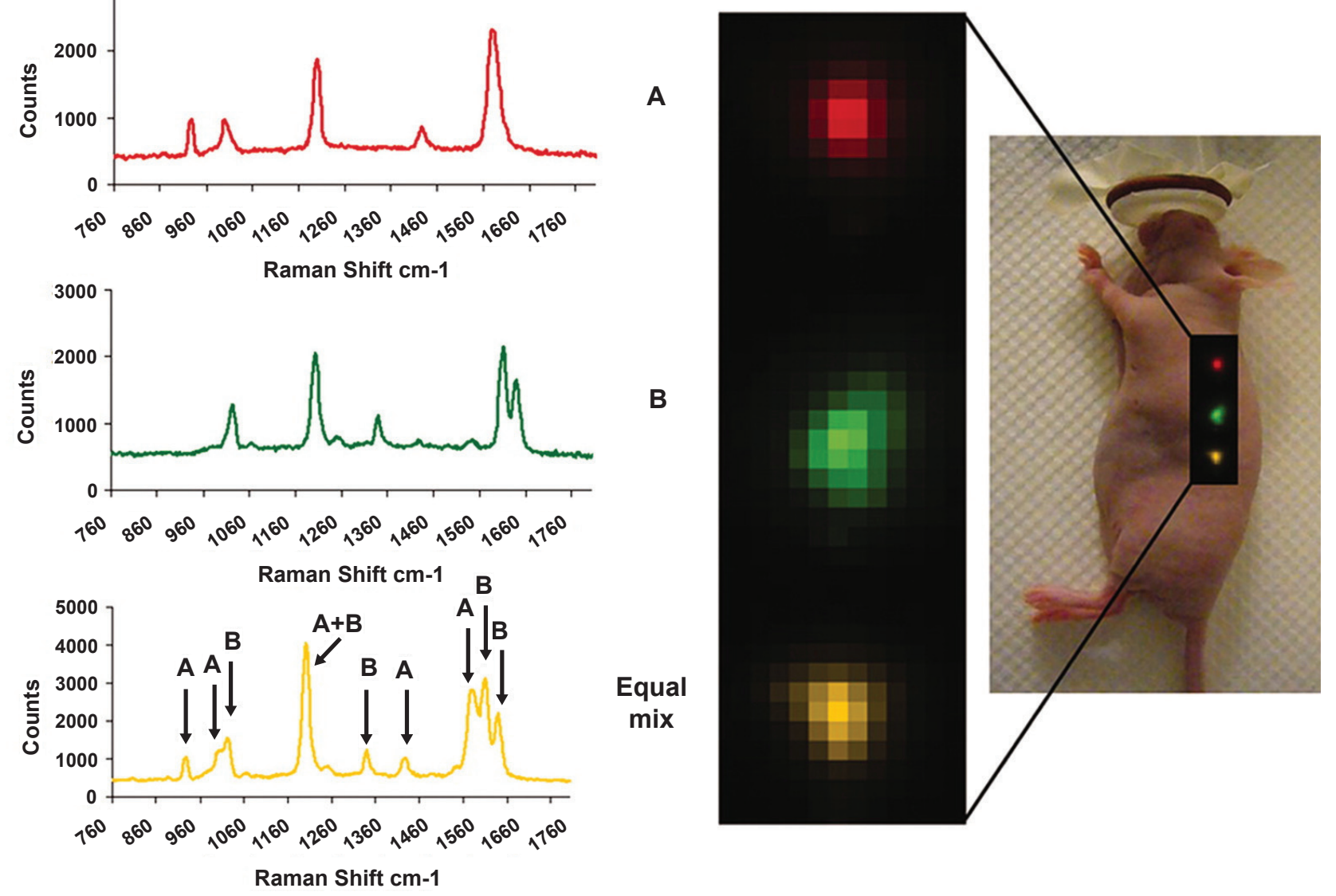

Equal mix

Figure 5 Multiplexed in vivo Raman imaging using SERS nanoparticles. Copyright @ 2008, PNAS. Adapted with permission from Keren S, Zavaleta C, Cheng Z, et al. 2008. Noninvasive molecular imaging of small living subjects using Raman spectroscopy. Proc Natl Acad Sci U SA, 105:5844-9.

NIR region, which can allow for multiplexed imaging of many tumor markers simultaneously if efficient targeting can be achieved, may have significant potential clinical applications. The imaging instruments used in these two studies are noncommercial prototype systems. Much future improvement in both the imaging system and fabrication/ modification of SERS nanoparticles will be needed before Raman imaging can become a clinical reality.

\section{Cancer therapy}

Conventional strategies for cancer intervention include surgery, chemotherapy, and radiation therapy. Taking advantage of their unique properties, most studies of gold nanoparticle-based cancer therapy have used photothermal therapy for the destruction of cancer cells or tumor tissue, which may be potentially useful in the clinical setting. When irradiated with focused laser pulses of suitable wavelength, targeted gold nanospheres, nanorods, nanoshells, and nanocages can kill bacteria (Zharov et al 2006b) and cancer cells (Loo et al 2005b; Huang et al 2006a, 2006b, 2007c; Chen et al 2007a; Tong et al 2007). It was estimated that
$70-80{ }^{\circ} \mathrm{C}$ was achieved through light absorption by the gold nanoparticles (Huang et al 2006b) and up to 150 antibodies can be conjugated to a nanoshell through a bifunctional PEG linker (Lowery et al 2006). One intriguing observation is that most of these studies targeted either EGFR or human epidermal growth factor receptor 2 (HER2), obviously due to the ready availability of monoclonal antibodies (already approved by the Food and Drug Administration [FDA] for cancer therapy) that recognize these two proteins.

Since the absorbance wavelength (in the visible range) of small gold nanospheres is not optimal for in vivo applications, the assembly of gold nanoclusters on the cell membrane was investigated (Zharov et al 2005). It was found that the formation of nanoclusters led to increased local absorption and red-shifting, compared to cells that did not have nanoclusters. Significant enhancement in laser-induced cancer cell killing was observed using an NIR laser. Gold nanoshells are sufficiently large (about $100-300 \mathrm{~nm}$ in diameter) to have SPR peaks in the NIR region. In one pioneering study, human breast carcinoma cells incubated with gold nanoshells were found to undergo photothermally induced morbidity 
upon exposure to NIR light (Figure 6) (Hirsch et al 2003b). In vivo testing revealed that exposure to low dose NIR light in solid tumors treated with gold nanoshells resulted in significant average temperature increase, capable of inducing irreversible tissue damage, while the controls (not treated with nanoshells) exhibited much lower average temperature when exposed to NIR light and appeared undamaged (Hirsch et al 2003b).

In a recent report, it was suggested that 5000 gold nanoshells per prostate cancer cell was needed to achieve cell kill (Stern et al 2007). PEG-coated nanoshells with peak absorption in the NIR region were intravenously injected into tumor-bearing nude mice (O'Neal et al 2004; Stern et al 2008). In one study, all tumors treated with the NIR laser were ablated and the mice appeared tumor free for several months while tumors in control animals (NIR laser treatment without nanoshell injection) continued to grow. In another study, $93 \%$ of tumor necrosis and regression was observed in a high dose nanoshell $(8.5 \mu \mathrm{L} / \mathrm{g})$ treated group (Stern et al 2008). Surprisingly, a slightly lower nanoshell dose $(7.0 \mu \mathrm{L} / \mathrm{g})$ only resulted in tumor growth arrest at 21 days but not tumor ablation. The reason why such a subtle difference in nanoshell dose could cause dramatically different therapeutic efficacy deserves careful investigation. It is worth noting that all these in vivo cancer therapy studies only involve passive tumor targeting but not specific molecular targeting. Passive tumor targeting is due to the non-specific accumulation of the nanoshells in the tumor, termed "the enhanced permeability and retention (EPR) effect", since the tumor vasculature is usually more leaky than normal blood vessels and there is no lymphatic drainage in the tumor (Maeda et al 2000).

The recruitment of monocytes into hypoxic regions within tumors has been exploited for photo-induced cell killing with gold nanoshells (Choi et al 2007). Besides photothermal therapy, gold nanoparticles have also been investigated in other therapeutic studies. Phthalocyanine (a photosensitizer) stabilized gold nanospheres (2-4 nm in diameter) have been reported for photodynamic therapy of cultured tumor cells (Wieder et al 2006). Gold nanoparticles have been shown to enhance the antiproliferation and apoptosis of human hepatoma cells induced by Paclitaxel, a chemotherapeutic drug (Wei et al 2007). A recent study has shown that enhancement

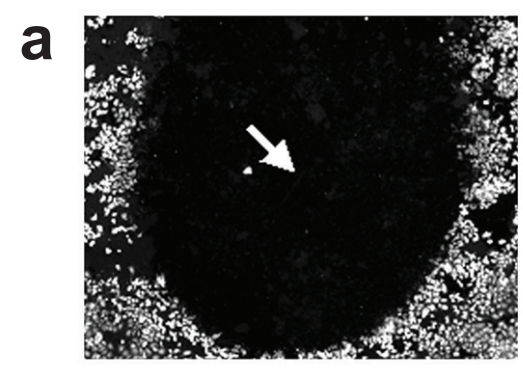

In vitro

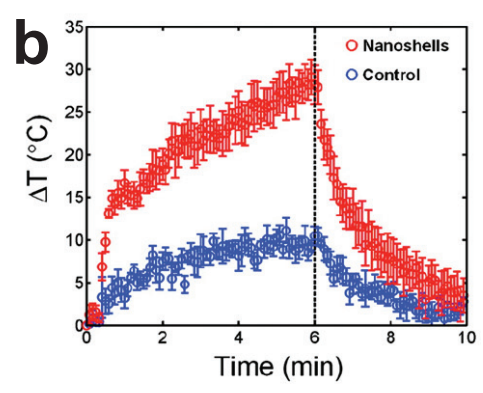

$2.5 \mathrm{~mm}$

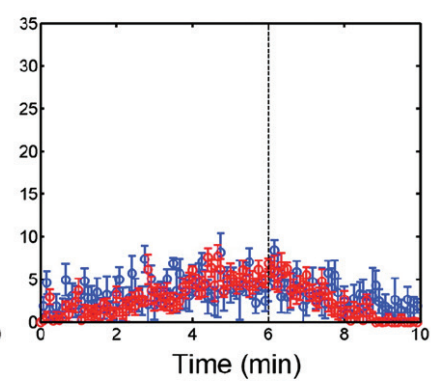

$7.3 \mathrm{~mm}$

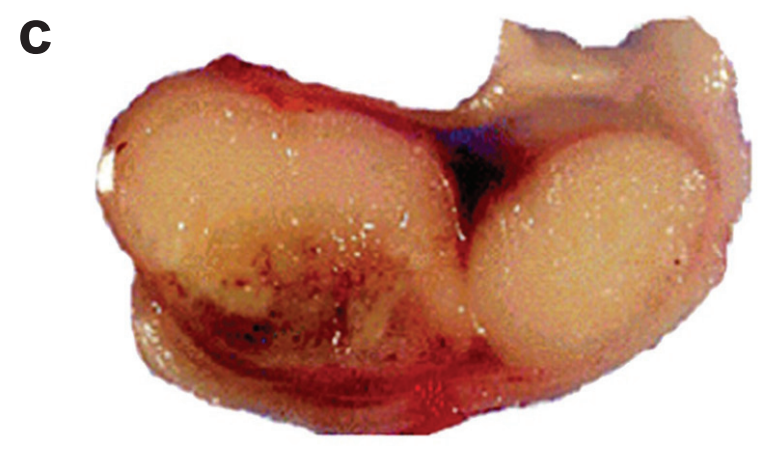

Tissue

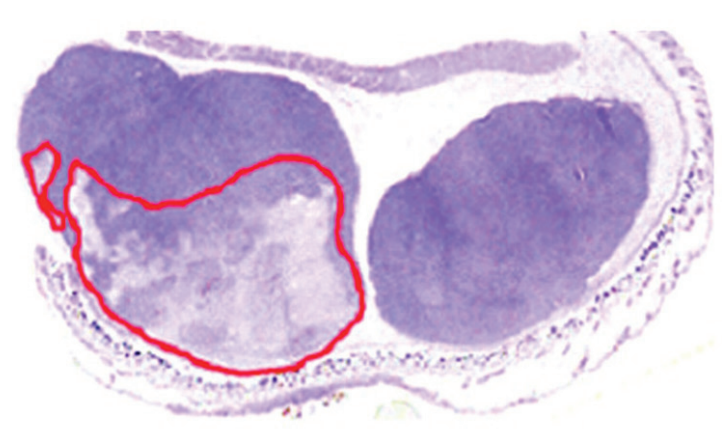

H\&E staining

Figure 6 Gold nanoshells can destroy cancer cells both in vitro and in vivo. a. Cells incubated with gold nanoshells can be killed by NIR light (dark area). b. Temporal plots of maximum temperature change of NIR-irradiated tumors with and without nanoshells at depths of $2.5 \mathrm{~mm}$ and $7.3 \mathrm{~mm}$ beneath the tissue surface. c. Gross pathology after in vivo treatment with nanoshells and NIR laser revealed hemorrhaging and loss of tissue birefringence beneath the apical tissue surface. Hematoxylin/eosin (H\&E) staining within the same plane confirms tissue damage within the area that contains nanoshells. Copyright @ 2008, PNAS. Adapted with permission from Hirsch LR, Stafford RJ, Bankson JA, et al. 2003b. Nanoshell-mediated near-infrared thermal therapy of tumors under magnetic resonance guidance. Proc Natl Acad Sci U SA, I00: I3549-54. 
of radiosensitivity can be achieved due to the increased absorption of ionizing radiation by the gold nanoparticles, which in turn caused breaks in single- and double-stranded DNA (Zheng et al 2008). Although it was proposed that targeting the DNA of cancer cells with gold nanoparticles may offer a novel approach that is generally applicable to external beam radiotherapy treatments, achieving DNA targeting in vivo is extremely difficult.

\section{Drug delivery}

Several studies have reported the use of gold nanoparticle as drug delivery vehicles. Tumor necrosis factor-alpha (TNF- $\alpha$ ), a cytokine with excellent anticancer efficacy, is systemically toxic which severely limited its therapeutic applications (van Horssen et al 2006; Mocellin and Nitti 2008). A nanoparticle delivery system, consisting of PEG coated gold nanoparticle loaded with TNF- $\alpha$, was constructed to maximize the tumor damage and minimize the systemic toxicity of TNF- $\alpha$ (Visaria et al 2006). Combination of local heating and nanoparticle-based delivery of TNF- $\alpha$ resulted in enhanced therapeutic efficacy than either treatment alone. Thermally-induced tumor growth delay was enhanced by pretreatment with the nanoparticle, when given intravenously at the proper dosage and timing. Tumor blood flow suppression, as well as tumor perfusion defects, suggested vascular damage-mediated tumor cell killing. Surprisingly, following intravenous administration, little to no accumulation in the RES (eg, liver and spleen) or other healthy organs of the animals was observed (Paciotti et al 2004). Subsequently, this nanoparticle conjugate has also been used to destroy the tumor within an iceball, again without significant systemic toxicity (Goel et al 2007). Phase I clinical trials of this conjugate, subsequently termed "CYT-6091" (Visaria et al 2007), are currently ongoing to evaluate its safety, pharmacokinetics, and clinical efficacy.

Methotrexate (MTX), an inhibitor of dihydrofolate reductase, is a chemotherapeutic agent for treating a variety of cancers types (Huennekens 1994). MTX-gold nanoparticle conjugate was prepared and the cytotoxic/antitumor effect was examined in vitro and in vivo (Chen et al 2007b). Administration of the conjugate suppressed tumor growth in a mouse model of Lewis lung carcinoma, whereas an equal dose of free MTX had no antitumor effect.

Nanoshells have been tested for drug delivery. In one early study, composites of hydrogels and gold nanoshells were developed for photothermally-modulated drug delivery (Sershen et al 2000). Irradiation at $1064 \mathrm{~nm}$ was absorbed by the nanoshells and converted to heat, which led to the collapse of the hydrogel thus significantly enhancing the drug release. Subsequently, modulated drug delivery of methylene blue, insulin, and lysozyme was achieved by irradiation of the drug-loaded nanoshell-hydrogel composites, with the drug release rate dependent upon the molecular weight of the therapeutic molecule (Bikram et al 2007). Hollow gold nanoshells can also encapsulate enzymes such as horseradish peroxidase (HRP), which remained active inside the nanoshells for small, but not large, substrate molecules (Kumar et al 2005). Not surprisingly, HRP did not show any activity when trapped inside solid gold nanoparticles.

Drug delivery using gold nanoparticles, in combination with their intrinsic capability for photothermal therapy, should be explored in the future. Currently, which type of gold nanoparticle is the most suitable for drug delivery applications is still debatable. It was found that the intracellular uptake of different sized and shaped gold nanoparticles are highly dependent upon their physical dimensions (Chithrani et al 2006). The absorption/scattering efficiency and optical resonance wavelengths have been calculated for three commonly used classes of gold nanoparticles: nanospheres, nanoshells, and nanorods (Jain et al 2006a). The narrow range in the SPR peaks of nanospheres $(\sim 520-550 \mathrm{~nm})$ resulted in very limited use for in vivo applications. The SPR peaks of gold nanoshells lie favorably in the NIR region. The total extinction of nanoshells has a linear dependence on the overall size, however independent of the core/shell radius ratio. The relative scattering contribution to the extinction can be rapidly increased by increasing the nanoshell size or decreasing the ratio of the core/shell radius. Gold nanorods were found to have comparable optical properties at much smaller effective size, with absorption and scattering coefficients an order of magnitude higher than those for nanoshells and nanospheres. While nanorod with a higher aspect ratio and a smaller effective radius is a better photoabsorbing nanoparticle suitable for therapeutic applications, that with a larger effective radius is more favorable for imaging purposes.

Studies have shown that femtosecond pulse excitation (at $400 \mathrm{~nm}$ wavelength) of DNA-modified nanoparticles can lead to desorption of the thiolated DNA strands from the nanoparticle surface by breaking the gold-sulfur bond (Jain et al 2006b). This property could be exploited in the future for controlled drug release. The stability of gold nanoparticle bioconjugates in high ionic strength media has been characterized as a function of the nanoparticle size, PEG length, and the monolayer composition (Liu et al 2007a). It was found that nanoparticle stability increased with increasing PEG length, decreasing nanoparticle diameter, and increasing 
PEG mole fraction. Importantly, gold nanoparticles modified with PEG chains of molecular weight (MW) 5000 were internalized as efficiently as analogous conjugates with PEG chains of MW 900. Based on this finding, gold nanoparticles functionalized with optimal-sized PEG chains (at least of MW 5000 to efficiently bypass the RES), with circulation half-life of at least a few hours, may be the most efficacious for cancer therapy.

In order to make gold nanoparticles more useful for drug delivery and other biomedical applications (imaging and therapy), they need to be effectively, specifically, and reliably directed to a specific organ or disease site without alteration. Specific targeting in vivo has not been achieved for gold nanoparticle-base drug delivery, due to the relatively large overall size of the conjugate (typically more than $50 \mathrm{~nm}$ in diameter) which prohibits efficient extravasation. Although utilization of passive targeting only has been shown to be efficacious in certain xenograft subcutaneous tumor models, they may not truly reflect the clinical situation. Transgenic and orthotopic tumor models are more clinically relevant and these tumors typically have much less leaky vasculature than subcutaneous ones, which will make passive targeting unsuitable for either cancer imaging or therapy. Molecular cancer markers over-expressed on the tumor vasculature may be the targets of choice.

\section{Summary and outlook}

Multifunctionality is the key advantage of nanoparticles over traditional approaches. Targeting ligands, imaging labels, therapeutic drugs, and many other functional moieties can all be integrated into the nanoparticle conjugate to allow for targeted molecular imaging and molecular therapy of cancer. Gold nanoparticle is unique in a sense because of its intriguing optical properties which can be exploited for both imaging and therapeutic applications (Figure 7). The future of nanomedicine lies in multifunctional nanoplatforms which combine both therapeutic components and multimodality imaging. The ultimate goal is that nanoparticle-based agents can allow for efficient, specific in vivo delivery of drugs without systemic toxicity, and the dose delivered as well as the therapeutic efficacy can be accurately measured noninvasively over time. Much remains to be done before this can be a clinical reality and many factors need to be optimized simultaneously for the best clinical outcome.

The most promising applications of nanoparticlebased agents will be in cardiovascular medicine, where there is much less biological barrier for efficient delivery of nanoparticles, and in oncology, where the leaky tumor vasculature can allow for better tissue penetration than in normal organs/tissues. We have found that quantum dots do not extravasate from the tumor vasculature after intravenous injection (Cai et al 2006, 2007a). Given the fact that the gold nanoparticles are mostly much larger than quantum dots $(>50 \mathrm{~nm}$ vs $<25 \mathrm{~nm}$ ), it is likely that gold nanoparticles do not extravasate either. Since photothermal tumor ablation is useful only when the nanoparticles are sufficiently large $(\sim 100 \mathrm{~nm}$ in diameter), small gold nanopheres $(<10 \mathrm{~nm})$ are not ideal for this goal. However, smaller particles may allow for much better extravasation hence better tumor targeting efficacy. Nonetheless, for both large and small gold nanoparticles, vasculature targeting is perhaps the only route that is likely to succeed in the clinic.

Optical imaging using gold nanoparticles has very limited clinical future. Among all imaging modalities, no

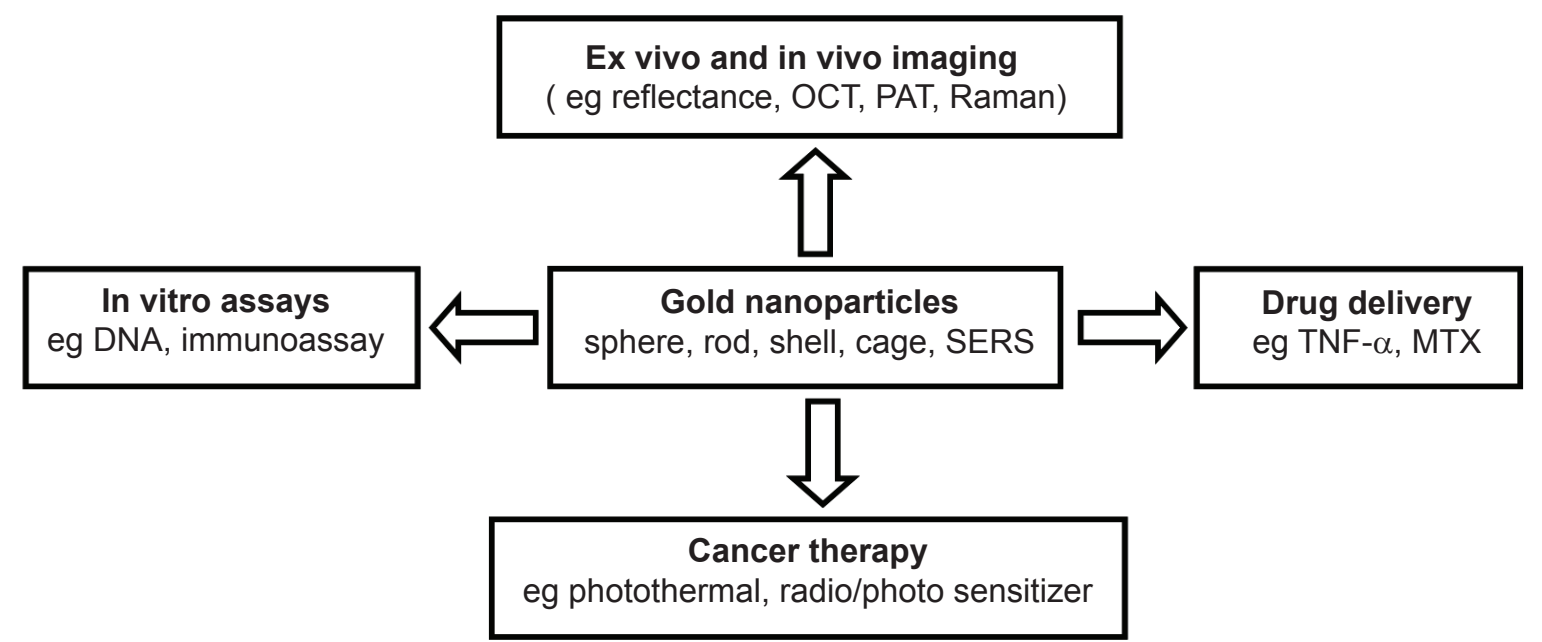

Figure 7 The versatile properties of gold nanoparticles have been employed for biomedical applications in many areas. 
single modality is perfect and sufficient to obtain all the necessary information for a particular question (Massoud and Gambhir 2003). For example, it is difficult to accurately quantify optical signal in living subjects, particularly in deep tissues; Radionuclide-based imaging techniques (eg, positron emission tomography [PET]), are very sensitive and highly quantitative but they have relatively poor spatial resolution. Combination of certain imaging modalities can offer synergistic advantages over any single modality alone (Cai and Chen 2007, 2008). Dual-modality agents that combine PET, which is very sensitive and highly quantitative (Phelps 2000), and optical imaging, which can significantly facilitate ex vivo validation of the in vivo data, should be of particular interest for future biomedical research. The relatively large size of the gold nanoparticle may potentially allow for simultaneous multiple receptor binding of the targeting ligands on the same particle. Thus, targeting multiple closely-related receptors simultaneously may be efficacious for targeted cancer imaging/therapy, with both improved sensitivity and better specificity. We envision that peptides or small molecules are better targeting ligands than antibodies for nanoparticle conjugation, not only to keep the overall size small but also to fully take advantage of the polyvalency effect (Mammen et al 1998) since significantly more small molecules $(\mathrm{MW}<1,000)$ can be attached to a nanoparticle than the macromolecular antibodies (MW 150,000). A multifunctional platform based on gold nanoparticles ("mix-and-match" with suitably selected components for each individual application) may provide the ultimate "magic gold bullet" for cancer intervention (Figure 8).

Nanoparticle-based ex vivo sensors and in vivo imaging are both critical for future optimization of cancer patient management. Ex vivo diagnostics (using blood samples drawn before, during, and after treatment) in combination

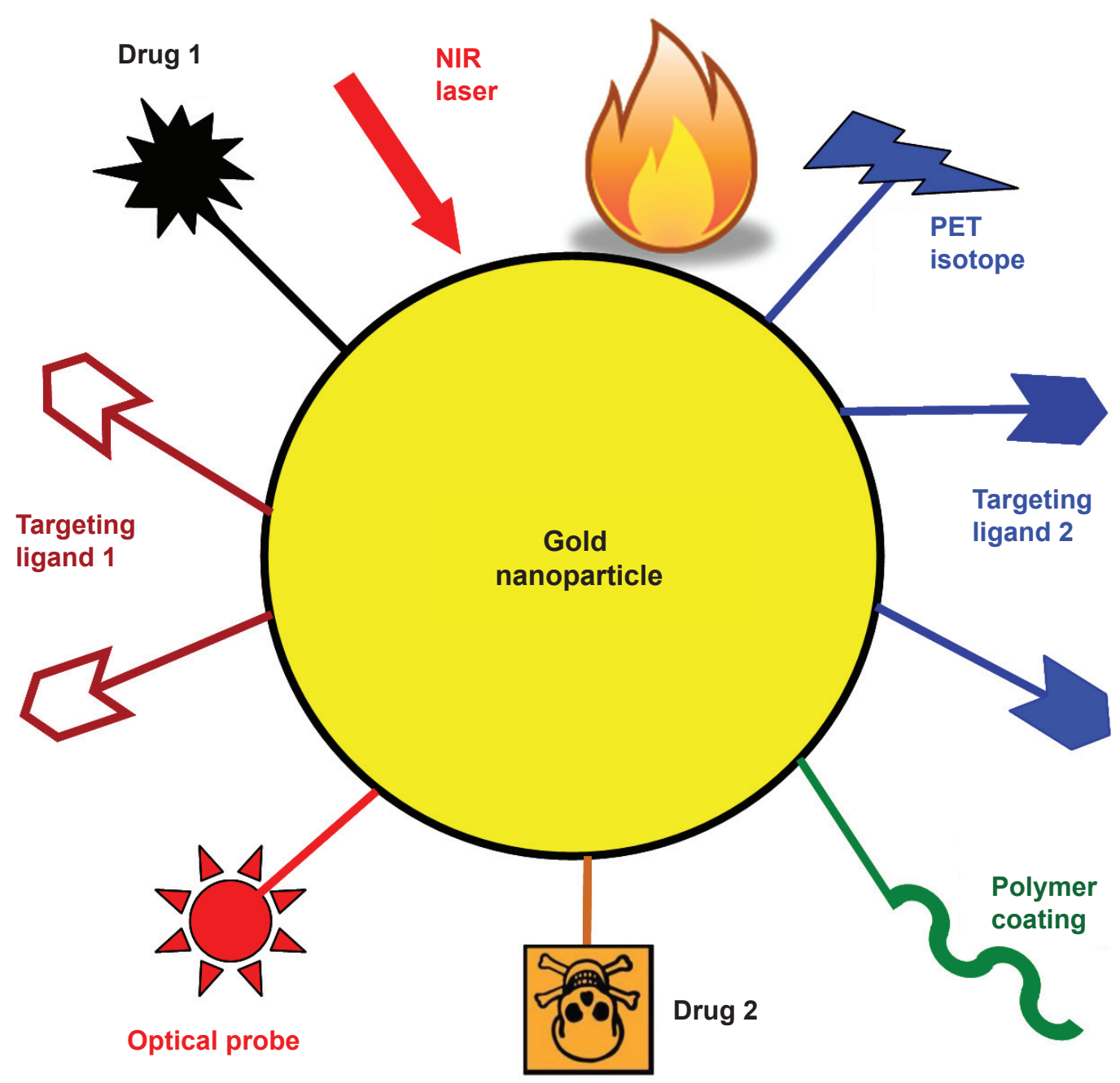

Figure $8 \mathrm{~A}$ multifunctional gold nanoparticle-based platform incorporating multiple receptor targeting, multimodality imaging, and multiple therapeutic entities. Not all functional moieties will be necessary and only suitably selected components are needed for each individual application. 
with in vivo diagnostics (noninvasive imaging before, during, and after treatment) can provide a synergistic approach that neither strategy alone can offer. Upon further development and validation, nanoparticle-based approaches (both ex vivo nanosensors and in vivo imaging) will eventually be able to predict which patients will likely respond to a specific molecular therapy and monitor their responses to personalized therapy. With the capacity to provide enormous sensitivity, throughput, and flexibility, nanotechnology has the potential to profoundly impact cancer diagnosis and patient management in the near future. Big strides have been made and many proof-of-principle studies have been successfully performed, the future looks brighter than ever yet many hurdles remain to be conquered.

\section{Acknowledgments}

Ting Gao acknowledges the support from Tyco Electronics.

\section{References}

Agrawal A, Huang S, Wei Haw Lin A, et al. 2006. Quantitative evaluation of optical coherence tomography signal enhancement with gold nanoshells. J Biomed Opt, 11:041121.

Anshup A, Venkataraman JS, Subramaniam C, et al. 2005. Growth of gold nanoparticles in human cells. Langmuir, 21:11562-7.

Bikram M, Gobin AM, Whitmire RE, et al. 2007. Temperature-sensitive hydrogels with $\mathrm{SiO} 2-\mathrm{Au}$ nanoshells for controlled drug delivery. J Control Release, 123:219-27.

Bremer C, Tung CH, Weissleder R. 2001. In vivo molecular target assessment of matrix metalloproteinase inhibition. Nat Med, 7:743-8.

Brust M, Walker M, Bethell D, et al. 1994. Synthesis of thiol-derivatized gold nanoparticles in a two-phase liquid-liquid system. $J$ Chem Soc Chem Commun, 801-2.

Busbee BD, Obare SO, Murphy CJ. 2003. An improved synthesis of highaspect-ratio gold nanorods. Adv Mater, 15:414-6.

Cai W, Chen K, Li ZB, et al. 2007a. Dual-function probe for PET and near-infrared fluorescence imaging of tumor vasculature. $J \mathrm{Nucl} \mathrm{Med}$, 48:1862-70.

Cai W, Chen X. 2007. Nanoplatforms for targeted molecular imaging in living subjects. Small, 3:1840-54.

Cai W, Chen X. 2008. Multimodality imaging of tumor angiogenesis. J Nucl Med, 49:113S-28S.

Cai W, Hsu AR, Li ZB, et al. 2007b. Are quantum dots ready for in vivo imaging in human subjects? Nanoscale Res Lett, 2:265-81.

Cai W, Shin DW, Chen K, et al. 2006. Peptide-labeled near-infrared quantum dots for imaging tumor vasculature in living subjects. Nano Lett, 6:669-76.

Cang H, Sun T, Li ZY, et al. 2005. Gold nanocages as contrast agents for spectroscopic optical coherence tomography. Opt Lett, 30:3048-50.

Canizal G, Ascencio JA, Gardea-Torresday J, et al. 2001. Multiple twinned gold nanorods grown by bio-reduction techniques. $J$ Nanopart Res, $3: 475-81$.

Cao YC, Jin R, Mirkin CA. 2002. Nanoparticles with Raman spectroscopic fingerprints for DNA and RNA detection. Science, 297:1536-40.

Caruso F, Spasova M, Salgueirino-Maceira V, et al. 2001. Multilayer assemblies of silica-encapsulated gold nanoparticles on decomposable colloid templates. Adv Mater, 13:1090-4.

Chang SS, Shih CW, Chen CD, et al. 1999. The shape transition of gold nanorods. Langmuir, 15:701-9.
Chen J, McLellan JM, Siekkinen A, et al. 2006. Facile synthesis of gold-silver nanocages with controllable pores on the surface. $J \mathrm{Am}$ Chem Soc, 128:14776-7.

Chen J, Saeki F, Wiley BJ, et al. 2005. Gold nanocages: bioconjugation and their potential use as optical imaging contrast agents. Nano Lett, 5:473-7.

Chen J, Wang D, Xi J, et al. 2007a. Immuno gold nanocages with tailored optical properties for targeted photothermal destruction of cancer cells. Nano Lett, 7:1318-22.

Chen YH, Tsai CY, Huang PY, et al. 2007b. Methotrexate conjugated to gold nanoparticles inhibits tumor growth in a syngeneic lung tumor model. Mol Pharm, 4:713-22.

Chithrani BD, Ghazani AA, Chan WC. 2006. Determining the size and shape dependence of gold nanoparticle uptake into mammalian cells. Nano Lett, 6:662-8.

Choi MR, Stanton-Maxey KJ, Stanley JK, et al. 2007. A cellular Trojan horse for delivery of therapeutic nanoparticles into tumors. Nano Lett, 7:3759-65.

Cognet L, Tardin C, Boyer D, et al. 2003. Single metallic nanoparticle imaging for protein detection in cells. Proc Natl Acad Sci U S A, 100:11350-5.

de la Fuente JM, Berry CC, Riehle MO, et al. 2006. Nanoparticle targeting at cells. Langmuir, 22:3286-93.

de Roos A, Doornbos J, Baleriaux D, et al. 1988. Clinical applications of gadolinium-DTPA in MRI. Magn Reson Annu, 113-45.

Dunn AR, Spudich JA. 2007. Dynamics of the unbound head during myosin V processive translocation. Nat Struct Mol Biol, 14:246-8.

Durr NJ, Larson T, Smith DK, et al. 2007. Two-photon luminescence imaging of cancer cells using molecularly targeted gold nanorods. Nano Lett, 7:941-5.

Esumi K, Suzuki A, Aihara N, et al. 1998. Preparation of gold colloids with UV irradiation using dendrimers as stabilizer. Langmuir, 14:3157-9.

Ferrari M. 2005. Cancer nanotechnology: opportunities and challenges. Nat Rev Cancer, 5:161-71.

Frangioni JV. 2003. In vivo near-infrared fluorescence imaging. Curr Opin Chem Biol, 7:626-34.

Frens G. 1973. Controlled nucleation for the regulation of the particle size in monodisperse gold suspensions. Nature, 241:20-2.

Gao X, Cui Y, Levenson RM, et al. 2004. In vivo cancer targeting and imaging with semiconductor quantum dots. Nat Biotechnol, 22:969-76.

Garcia ME, Baker LA, Crooks RM. 1999. Preparation and characterization of dendrimer-gold colloid nanocomposites. Anal Chem, 71:256-8.

Giersig M, Mulvaney P. 1993. Preparation of ordered colloid monolayers by electrophoretic deposition. Langmuir, 9:3408-13.

Goel R, Swanlund D, Coad J, et al. 2007. TNF-alpha-based accentuation in cryoinjury - dose, delivery, and response. Mol Cancer Ther, 6:2039-47.

Grodzinski P, Silver M, Molnar LK. 2006. Nanotechnology for cancer diagnostics: promises and challenges. Expert Rev Mol Diagn, 6:307-18.

Han S, Lin J, Zhou F, et al. 2000. Oligonucleotide-capped gold nanoparticles for improved atomic force microscopic imaging and enhanced selectivity in polynucleotide detection. Biochem Biophys Res Commun, 279:265-9.

Hauck TS, Ghazani AA, Chan WC. 2008. Assessing the effect of surface chemistry on gold nanorod uptake, toxicity, and gene expression in mammalian cells. Small, 4:153-9.

Hering K, Cialla D, Ackermann K, et al. 2008. SERS: a versatile tool in chemical and biochemical diagnostics. Anal Bioanal Chem, 390:113-24.

Hiramatsu H, Osterloh FE. 2004. A simple large-scale synthesis of nearly monodisperse gold and silver nanoparticles with adjustable sizes and with exchangeable surfactants. Chem Mater, 16:2509-11.

Hirsch LR, Halas NJ, West JL. 2005. Whole-blood immunoassay facilitated by gold nanoshell-conjugate antibodies. Methods Mol Biol, 303:101-11.

Hirsch LR, Jackson JB, Lee A, et al. 2003a. A whole blood immunoassay using gold nanoshells. Anal Chem, 75:2377-81. 
Hirsch LR, Stafford RJ, Bankson JA, et al. 2003b. Nanoshell-mediated near-infrared thermal therapy of tumors under magnetic resonance guidance. Proc Natl Acad Sci U S A, 100:13549-54.

Horton MA, Khan A. 2006. Medical nanotechnology in the UK: a perspective from the London Centre for Nanotechnology. Nanomedicine, $2: 42-8$.

Huang X, El-Sayed IH, Qian W, et al. 2006a. Cancer cell imaging and photothermal therapy in the near-infrared region by using gold nanorods. $J$ Am Chem Soc, 128:2115-20.

Huang X, El-Sayed IH, Qian W, et al. 2007a. Cancer cells assemble and align gold nanorods conjugated to antibodies to produce highly enhanced, sharp, and polarized surface Raman spectra: a potential cancer diagnostic marker. Nano Lett, 7:1591-7.

Huang X, Jain PK, El-Sayed IH, et al. 2006b. Determination of the minimum temperature required for selective photothermal destruction of cancer cells with the use of immunotargeted gold nanoparticles. Photochem Photobiol, 82:412-17.

Huang X, Jain PK, El-Sayed IH, et al. 2007b. Gold nanoparticles: interesting optical properties and recent applications in cancer diagnostics and therapy. Nanomed, 2:681-93.

Huang X, Qian W, El-Sayed IH, et al. 2007c. The potential use of the enhanced nonlinear properties of gold nanospheres in photothermal cancer therapy. Lasers Surg Med, 39:747-53.

Huennekens FM. 1994. The methotrexate story: a paradigm for development of cancer chemotherapeutic agents. Adv Enzyme Regul, 34:397-419.

Intes X. 2005. Time-domain optical mammography SoftScan: initial results. Acad Radiol, 12:934-47.

Jaffer FA, Kim DE, Quinti L, et al. 2007. Optical visualization of cathepsin $\mathrm{K}$ activity in atherosclerosis with a novel, protease-activatable fluorescence sensor. Circulation, 115:2292-8.

Jain PK, Lee KS, El-Sayed IH, et al. 2006a. Calculated absorption and scattering properties of gold nanoparticles of different size, shape, and composition: applications in biological imaging and biomedicine. J Phys Chem B, 110:7238-48.

Jain PK, Qian W, and El-Sayed MA. 2006b. Ultrafast cooling of photoexcited electrons in gold nanoparticle-thiolated DNA conjugates involves the dissociation of the gold-thiol bond. $J$ Am Chem Soc, 128:2426-33

Jana NR, Gearheart L, Murphy CJ. 2001a. Seed-mediated growth approach for shape-controlled synthesis of spheroidal and rod-like gold nanoparticles using a surfactant template. Adv Mater, 13:1389-93.

Jana NR, Gearheart L, and Murphy CJ. 2001b. Wet chemical synthesis of high aspect ratio cylindrical gold nanorods. J Phys Chem B, 105:4065-7.

Jana NR, Gearheart L, Obare SO, et al. 2002. Anisotropic chemical reactivity of gold spheroids and nanorods. Langmuir, 18:922-7.

Jemal A, Siegel R, Ward E, et al. 2008. Cancer statistics, 2008. CA Cancer J Clin, 58:71-96.

Jensen RA, Sherin J, Emory SR. 2007. Single nanoparticle based optical pH probe. Appl Spectrosc, 61:832-8.

Jin R, He X, Wang K, et al. 2007. Characterization of different sequences of DNA on si substrate by atomic force microscopy and gold nanoparticle labeling. J Nanosci Nanotechnol, 7:418-23.

Kah JC, Kho KW, Lee CG, et al. 2007. Early diagnosis of oral cancer based on the surface plasmon resonance of gold nanoparticles. Int J Nanomedicine, 2:785-98.

Kawasaki ES, Player A. 2005. Nanotechnology, nanomedicine, and the development of new, effective therapies for cancer. Nanomedicine, 1:101-9.

Keren S, Zavaleta C, Cheng Z, et al. 2008. Noninvasive molecular imaging of small living subjects using Raman spectroscopy. Proc Natl Acad Sci U S A, 105:5844-9.

Kim F, Song JH, Yang P. 2002. Photochemical synthesis of gold nanorods. $J$ Am Chem Soc, 124:14316-7.

Kim YG, Oh SK, Crooks RM. 2004. Preparation and characterization of 1-2 nm dendrimer-encapsulated gold nanoparticles having very narrow size distributions. Chem Mater, 16:167-72.
Kim YP, Oh E, Hong MY, et al. 2006. Gold nanoparticle-enhanced secondary ion mass spectrometry imaging of peptides on self-assembled monolayers. Anal Chem, 78:1913-20.

Kirchner C, Liedl T, Kudera S, et al. 2005. Cytotoxicity of colloidal CdSe and $\mathrm{CdSe} / \mathrm{ZnS}$ nanoparticles. Nano Lett, 5:331-8.

Kumar R, Maitra AN, Patanjali PK, et al. 2005. Hollow gold nanoparticles encapsulating horseradish peroxidase. Biomaterials, 26:6743-53.

Lal S, Grady NK, Goodrich GP, et al. 2006. Profiling the near field of a plasmonic nanoparticle with Raman-based molecular rulers. Nano Lett, 6:2338-43.

Laurent G, Felidj N, Truong SL, et al. 2005. Imaging surface plasmon of gold nanoparticle arrays by far-field Raman scattering. Nano Lett, 5:253-8.

Lee S, Cha EJ, Park K, et al. 2008. A near-infrared-fluorescence-quenched gold-nanoparticle imaging probe for in vivo drug screening and protease activity determination. Angew Chem Int Ed Engl, 47:2804-7.

Leff DV, Brandt L, Heath JR. 1996. Synthesis and characterization of hydrophobic, organically soluble gold nanocrystals functionalized with primary amines. Langmuir, 12:4723-30.

Li PC, Wei CW, Liao CK, et al. 2007a. Photoacoustic imaging of multiple targets using gold nanorods. IEEE Trans Ultrason Ferroelectr Freq Control, 54:1642-7.

Li Y, Wark AW, Lee HJ, et al. 2006. Single-nucleotide polymorphism genotyping by nanoparticle-enhanced surface plasmon resonance imaging measurements of surface ligation reactions. Anal Chem, 78:3158-64.

Li ZB, Cai W, Chen X. 2007b. Semiconductor quantum dots for in vivo imaging. J Nanosci Nanotechnol, 7:2567-81.

Liu X, Dai Q, Austin L, et al. 2008. A one-step homogeneous immunoassay for cancer biomarker detection using gold nanoparticle probes coupled with dynamic light scattering. J Am Chem Soc, 130:2780-2.

Liu Y, Shipton MK, Ryan J, et al. 2007a. Synthesis, stability, and cellular internalization of gold nanoparticles containing mixed peptidepoly(ethylene glycol) monolayers. Anal Chem, 79:2221-9.

Liu Z, Cai W, He L, et al. 2007b. In vivo biodistribution and highly efficient tumour targeting of carbon nanotubes in mice. Nat Nanotechnol, 2:47-52.

Loo C, Hirsch L, Lee MH, et al. 2005a. Gold nanoshell bioconjugates for molecular imaging in living cells. Opt Lett, 30:1012-4.

Loo C, Lowery A, Halas N, et al. 2005b. Immunotargeted nanoshells for integrated cancer imaging and therapy. Nano Lett, 5:709-11.

Low AF, Tearney GJ, Bouma BE, et al. 2006. Technology Insight: optical coherence tomography - current status and future development. Nat Clin Pract Cardiovasc Med, 3:154-62; quiz 72.

Lowery AR, Gobin AM, Day ES, et al. 2006. Immunonanoshells for targeted photothermal ablation of tumor cells. Int J Nanomedicine, 1:149-54.

Maeda H, Wu J, Sawa T, et al. 2000. Tumor vascular permeability and the EPR effect in macromolecular therapeutics. A review. $J$ Control Release, 65:271-84.

Mammen M, Chio S, Whitesides GM. 1998. Polyvalent interactions in biological systems: implications for design and use of multivalent ligands and inhibitors. Angew Chem Int Ed Engl, 37:2755-94.

Manna A, Imae T, Aoi K, et al. 2001. Synthesis of dendrimer-passivated noble metal nanoparticles in a polar medium: comparison of size between silver and gold particles. Chem Mater, 13:1674-81.

Martin CR. 1994. Nanomaterials: a membrane-based synthetic approach. Science, 266:1961-6.

Massoud TF, Gambhir SS. 2003. Molecular imaging in living subjects: seeing fundamental biological processes in a new light. Genes Dev, 17:545-80.

Medley CD, Smith JE, Tang Z, et al. 2008. Gold nanoparticle-based colorimetric assay for the direct detection of cancerous cells. Anal Chem, 80:1067-72.

Michalet X, Pinaud FF, Bentolila LA, et al. 2005. Quantum dots for live cells, in vivo imaging, and diagnostics. Science, 307:538-44.

Mieszawska AJ, Zamborini FP. 2005. Gold nanorods grown directly on surfaces from microscale patterns of gold seeds. Chem Mater, 17:3415-20. 
Mocellin S, Nitti D. 2008. TNF and cancer: the two sides of the coin. Front Biosci, 13:2774-83.

Nitin N, Javier DJ, Richards-Kortum R. 2007a. Oligonucleotide-coated metallic nanoparticles as a flexible platform for molecular imaging agents. Bioconjug Chem, 18:2090-6.

Nitin N, Javier DJ, Roblyer DM, et al. 2007b. Widefield and high-resolution reflectance imaging of gold and silver nanospheres. $J$ Biomed Opt, 12:051505.

O'Neal DP, Hirsch LR, Halas NJ, et al. 2004. Photo-thermal tumor ablation in mice using near infrared-absorbing nanoparticles. Cancer Lett, 209:171-6.

Oldenburg SJ, Averitt RD, Westcott SL, et al. 1998. Nanoengineering of optical resonances. Chem Phys Lett, 288:243-7.

Oldenburg SJ, Jackson JB, Westcott SL, et al. 1999a. Infrared extinction properties of gold nanoshells. Appl Phys Lett, 75:2897-9.

Oldenburg SJ, Westcott SL, Averitt RD, et al. 1999b. Surface enhanced Raman scattering in the near infrared using metal nanoshell substrates. J Chem Phys, 111:4729-35.

Oyelere AK, Chen PC, Huang X, et al. 2007. Peptide-conjugated gold nanorods for nuclear targeting. Bioconjug Chem, 18:1490-7.

Paciotti GF, Myer L, Weinreich D, et al. 2004. Colloidal gold: a novel nanoparticle vector for tumor directed drug delivery. Drug Deliv, 11:169-83.

Park JW, Benz CC, Martin FJ. 2004. Future directions of liposomeand immunoliposome-based cancer therapeutics. Semin Oncol, 31:196-205.

Parkin DM. 2001. Global cancer statistics in the year 2000. Lancet Oncol, 2:533-43.

Parkin DM, Pisani P, Ferlay J. 1999. Estimates of the worldwide incidence of 25 major cancers in 1990. Int J Cancer, 80:827-41.

Peleg G, Lewis A, Linial M, et al. 1999. Nonlinear optical measurement of membrane potential around single molecules at selected cellular sites. Proc Natl Acad Sci U S A, 96:6700-4.

Phelps ME. 2000. PET: the merging of biology and imaging into molecular imaging. J Nucl Med, 41:661-81.

Pisani P, Parkin DM, Bray F, et al. 1999. Estimates of the worldwide mortality from 25 cancers in 1990. Int J Cancer, 83:18-29.

Qian X, Peng XH, Ansari DO, et al. 2008. In vivo tumor targeting and spectroscopic detection with surface-enhanced Raman nanoparticle tags. Nat Biotechnol, 26:83-90.

Qin WJ, Yung LY. 2007. Nanoparticle-based detection and quantification of DNA with single nucleotide polymorphism (SNP) discrimination selectivity. Nucleic Acids Res, 35:e111.

Radloff C, Vaia RA, Brunton J, et al. 2005. Metal nanoshell assembly on a virus bioscaffold. Nano Lett, 5:1187-91.

Reetz MT, Helbig W. 1994. Size-selective synthesis of nanostructured transition metal clusters. $J$ Am Chem Soc, 116:7401-2.

Sahoo SK, Parveen S, Panda JJ. 2007. The present and future of nanotechnology in human health care. Nanomedicine, 3:20-31.

Scott RWJ, Wilson OM, Crooks RM. 2005. Synthesis, characterization, and applications of dendrimer-encapsulated nanoparticles. $J$ Phys Chem B, 109:692-704.

Sershen SR, Westcott SL, Halas NJ, et al. 2000. Temperature-sensitive polymer-nanoshell composites for photothermally modulated drug delivery. J Biomed Mater Res, 51:293-8.

Sha MY, Xu H, Penn SG, et al. 2007. SERS nanoparticles: a new optical detection modality for cancer diagnosis. Nanomed, 2:725-34.

Shah K, Tung CH, Chang CH, et al. 2004. In vivo imaging of HIV protease activity in amplicon vector-transduced gliomas. Cancer Res, 64:273-8.

Shi X, Ganser TR, Sun K, et al. 2006. Characterization of crystalline dendrimer-stabilized gold nanoparticles. Nanotechnology, 17:1072-8.

Shi X, Lee I, Baker JR. 2008. Acetylation of dendrimer-entrapped gold and silver nanoparticles. J Mater Chem, 18:586-93.

Shi X, Wang S, Meshinchi S, et al. 2007a. Dendrimer-entrapped gold nanoparticles as a platform for cancer-cell targeting and imaging. Small, 3:1245-52.
Shi X, Wang S, Sun H, et al. 2007b. Improved biocompatibility of surface functionalized dendrimer-entrapped gold nanoparticles. Soft Matter, 3:71-4.

Sipkins DA, Cheresh DA, Kazemi MR, et al. 1998. Detection of tumor angiogenesis in vivo by $\alpha_{v} \beta_{3}$-targeted magnetic resonance imaging. Nat Med, 4:623-6.

Sokolov K, Follen M, Aaron J, et al. 2003. Real-time vital optical imaging of precancer using anti-epidermal growth factor receptor antibodies conjugated to gold nanoparticles. Cancer Res, 63:1999-2004.

Son SJ, Lee SB. 2007. A platform for ultrasensitive and selective multiplexed marker protein assay toward early-stage cancer diagnosis. Nanomed, 2:79-82.

Stern JM, Stanfield J, Kabbani W, et al. 2008. Selective prostate cancer thermal ablation with laser activated gold nanoshells. J Urol, 179:748-53.

Stern JM, Stanfield J, Lotan Y, et al. 2007. Efficacy of laser-activated gold nanoshells in ablating prostate cancer cells in vitro. J Endourol, 21:939-43.

$\mathrm{Su} \mathrm{CH}$, Sheu HS, Lin CY, et al. 2007. Nanoshell magnetic resonance imaging contrast agents. J Am Chem Soc, 129:2139-46.

Suzuki D, Kawaguchi H. 2005. Gold nanoparticle localization at the core surface by using thermosensitive core-shell particles as a template. Langmuir, 21:12016-24.

Tang D, Yuan R, Chai Y. 2007. Biochemical and immunochemical characterization of the antigen-antibody reaction on a non-toxic biomimetic interface immobilized red blood cells of crucian carp and gold nanoparticles. Biosens Bioelectron, 22:1116-20.

Taroni P, Danesini G, Torricelli A, et al. 2004. Clinical trial of time-resolved scanning optical mammography at 4 wavelengths between 683 and $975 \mathrm{~nm}$. J Biomed Opt, 9:464-73.

Thayer AM. 2007. Building up nanotech research. Chem Eng News, $85: 15-21$.

Thorek DL, Chen AK, Czupryna J, et al. 2006. Superparamagnetic iron oxide nanoparticle probes for molecular imaging. Ann Biomed Eng, 34:23-38.

Tong L, Zhao Y, Huff TB, et al. 2007. Gold nanorods mediate tumor cell death by compromising membrane integrity. Adv Mater, 19:3136-41.

Tseng WL, Huang MF, Huang YF, et al. 2005. Nanoparticle-filled capillary electrophoresis for the separation of long DNA molecules in the presence of hydrodynamic and electrokinetic forces. Electrophoresis, 26:3069-75.

Turkevich J, Stevenson PC, Hillier J. 1951. The nucleation and growth processes in the synthesis of colloidal gold. Discuss Faraday Soc, 11:55-75.

van der Zande BMI, Boehmer MR, Fokkink LGJ, et al. 1997. Aqueous gold sols and rod-shaped particles. J Phys Chem B, 101:852-4.

van Horssen R, Ten Hagen TL, Eggermont AM. 2006. TNF-alpha in cancer treatment: molecular insights, antitumor effects, and clinical utility. Oncologist, 11:397-408.

Visaria R, Bischof JC, Loren M, et al. 2007. Nanotherapeutics for enhancing thermal therapy of cancer. Int J Hyperthermia, 23:501-11.

Visaria RK, Griffin RJ, Williams BW, et al. 2006. Enhancement of tumor thermal therapy using gold nanoparticle-assisted tumor necrosis factoralpha delivery. Mol Cancer Ther, 5:1014-20.

Wang J, Zhu X, Tu Q, et al. 2008a. Capture of p53 by electrodes modified with consensus DNA duplexes and amplified voltammetric detection using ferrocene-capped gold nanoparticle/streptavidin conjugates. Anal Chem, 80:769-74.

Wang Y, Qian W, Tan Y, et al. 2008b. A label-free biosensor based on gold nanoshell monolayers for monitoring biomolecular interactions in diluted whole blood. Biosens Bioelectron, 23:1166-70.

Weare WW, Reed SM, Warner MG, et al. 2000. Improved synthesis of small (dCORE 1.5 nm) phosphine-stabilized gold nanoparticles. $J$ Am Chem Soc, 122:12890-1.

Wei XL, Mo ZH, Li B, et al. 2007. Disruption of HepG2 cell adhesion by gold nanoparticle and Paclitaxel disclosed by in situ QCM measurement. Colloids Surf B Biointerfaces, 59:100-4. 
Weissleder R, Tung CH, Mahmood U, et al. 1999. In vivo imaging of tumors with protease-activated near-infrared fluorescent probes. Nat Biotechnol, 17:375-8.

Wieder ME, Hone DC, Cook MJ, et al. 2006. Intracellular photodynamic therapy with photosensitizer-nanoparticle conjugates: cancer therapy using a 'Trojan horse'. Photochem Photobiol Sci, 5:727-34.

Xie H, Gill-Sharp KL, O’Neal DP. 2007. Quantitative estimation of gold nanoshell concentrations in whole blood using dynamic light scattering. Nanomedicine, 3:89-94.

Yang PH, Sun X, Chiu JF, et al. 2005. Transferrin-mediated gold nanoparticle cellular uptake. Bioconjug Chem, 16:494-6.

Yao J, Larson DR, Vishwasrao HD, et al. 2005. Blinking and nonradiant dark fraction of water-soluble quantum dots in aqueous solution. Proc Natl Acad Sci US A, 102:14284-9.

Yu YY, Chang SS, Lee CL, et al. 1997. Gold nanorods: electrochemical synthesis and optical properties. J Phys Chem B, 101:6661-4.

Yuan JJ, Schmid A, Armes SP, et al. 2006. Facile synthesis of highly biocompatible poly(2-(methacryloyloxy)ethyl phosphorylcholine)-coated gold nanoparticles in aqueous solution. Langmuir, 22:11022-7
Zhang J, Song S, Wang L, et al. 2007. A gold nanoparticle-based chronocoulometric DNA sensor for amplified detection of DNA. Nat Protoc, 2:2888-95.

Zharov VP, Galanzha EI, Shashkov EV, et al. 2006a. In vivo photoacoustic flow cytometry for monitoring of circulating single cancer cells and contrast agents. Opt Lett, 31:3623-5.

Zharov VP, Galitovskaya EN, Johnson C, et al. 2005. Synergistic enhancement of selective nanophotothermolysis with gold nanoclusters: potential for cancer therapy. Lasers Surg Med, 37:219-26.

Zharov VP, Mercer KE, Galitovskaya EN, et al. 2006b. Photothermal nanotherapeutics and nanodiagnostics for selective killing of bacteria targeted with gold nanoparticles. Biophys J, 90:619-27.

Zheng Y, Hunting DJ, Ayotte P, et al. 2008. Radiosensitization of DNA by gold nanoparticles irradiated with high-energy electrons. Radiat Res, 169:19-27. 